\title{
Comments to "Survey by the ANSM of the imaging protocol, detection rate, and safety of 68Ga-PSMA-11 PET/CT"
}

\author{
Francesco Giammarile ${ }^{1} \cdot$ Stefano Fanti ${ }^{2}$ \\ Published online: 19 March 2021 \\ (C) The Author(s), under exclusive licence to Springer-Verlag GmbH Germany, part of Springer Nature 2021
}

We read with interest the Article written by Yanna-Marina Chevalme and colleagues published in this issue of the EJNMMI [1]. Indeed, we agree with several considerations, first of all regarding the usefulness of collecting more data on the value of PSMA PET/CT.

However, there are other points that should be carefully considered, especially in view of the fact that the survey reported in the paper was promoted by a National Agency, namely the Agence Nationale de Securité du Médicament et des produits de santé (ANSM). In our field, we have already seen some examples of registry endorsed by regulatory authorities, the most famous being the National Oncologic PET Registry (NOPR) [2]. By means of NOPR, our US colleagues reported results addressing the impact of PET: they initially assessed the impact of PET with 18F-FDG, and a subsequent extension assessed the impact of $18 \mathrm{~F}$-fluoride PET [3]. In these cases, the study design was very clear: in fact, the objective was to study the intended management of patients with cancer using prospectively collected data obtained before and after PET.

In the present survey, the objective is completely different: to assess the added value of PSMA PET in the case of nonconclusive $18 \mathrm{~F}-\mathrm{FCH}$ PET. The difference is absolutely relevant, as the scope of the study has nothing to do with the clinical usefulness of the information provided by the new PET imaging, but rather to make a comparison with the existing approach in the same clinical scenario. Again, this is much understandable, as, for NOPR, there was no clear

This article is part of the Topical Collection on Oncology - Genitourinary

Stefano Fanti

Stefano.fanti@aosp.bo.it

1 Nuclear Medicine and Diagnostic Imaging Section, International Atomic Energy Agency, Vienna, Austria

2 Department of Nuclear Medicine, IRCCS Azienda Ospedaliero-Universitaria di Bologna, Medicina Nucleare, PAD 30 , Policlinico S.Orsola, Bologna, Italy comparator to $18 \mathrm{~F}-\mathrm{FDG}$ PET, while, for exploring biochemical recurrence (BCR) of prostate cancer, a tracer (18F-FCH) was available in 2016, when the ANSM promoted the survey. The first point really surprising for us was the choice of investigating the added value of the new tracer (68Ga-PSMA) only in patients whose $18 \mathrm{~F}-\mathrm{FCH} \mathrm{PET} / \mathrm{CT}$ was non-conclusive, meaning negative or equivocal. Apart from further consideration about the fact that a negative PET (i.e., without pathologic uptake) cannot necessarily be regarded as non-conclusive, the surprise was about the main objective of the study, as we would have expected a direct comparative study, aimed at establishing the superiority of one tracer over the other. Similar studies can be done for PET imaging [4] and thus, the decision of ANSM to grant a nominative authorization only in patients whose previous $18 \mathrm{~F}-\mathrm{FCH}$ PET/CT was nonconclusive sounds questionable to us, as such an approach would by design never allow us to show any superiority.

A further point of interest is about the used measure of diagnostic performance of 68Ga-PSMA PET/CT, namely the positivity rate (PR). The authors properly describe such a limitation in the discussion section, and we fully agree that a standard of truth is difficult to obtain in recurrent prostate cancer: nonetheless, most other studies have used composite standard to address at least detection rate (DR) rather than PR, while in the present manuscript, the detection rate is inexactly mentioned in the title but then never addressed in the study. It would have been much more robust for the study design to aim at determining DR, using correlative imaging, follow-up, or other data. The use of PR as the main objective of the study poses a relevant question about the hypothesis to evaluate: what if the new tracer produces a very high number of falsepositive findings? In such a case, the survey would have reported a very high PR and thus a very good added value over $18 \mathrm{~F}-\mathrm{FCH}$ PET, but such data would have been of no clinical use or even dangerous to apply.

With respect to enrolled patients, another curious aspect was the patient's characteristic of PSA: mean PSA values were $1.62 \mathrm{ng} / \mathrm{mL}$, with 356 cases having PSA $<1 \mathrm{ng} / \mathrm{mL}$. At 
the time of designing the study (2015), the most authoritative guideline for prostate cancer were endorsed by the European Association of Urology (EAU) and for BCR imaging with choline PET suggested a cutoff value between 1 and $2 \mathrm{ng} /$ $\mathrm{mL}$ : therefore, about $1 / 3$ of the $18 \mathrm{~F}-\mathrm{FCH}$ scans of the French study were done in patients not matching the recommendation of international guidelines, and 17 of them had a PSA $<0.2 \mathrm{ng} / \mathrm{mL}$, thus being even questionable regarding the BCR status. To keep a further eye on EAU guidelines, it is worth mentioning that since 2018, in the case of BCR and $\mathrm{PSA} \geq 1 \mathrm{ng} / \mathrm{mL}$, it is suggested to perform a PSMA PET/CT if available or a choline PET/CT imaging otherwise; it is clear that an international guideline has no binding value over protocols promoted by National Agencies, but essentially in the last part of the survey, it was running against guidelines indications.

We would like to make a final remark regarding the time elapsed: PSMA was introduced as a prostate cancer radiotracer in 2014, and the French Agency was very rapid at promoting the survey and that was much appreciable, leading to the start of PSMA scanning with nominative authorization as early as May 2016. However, the enrolment lasted almost 3 years, and it was not modified despite the market authorization granted by EMA to Fluciclovine in May 2017: the lack of any consideration for fluciclovine is indeed another weakness of the manuscript. It then took more than 1 year to elaborate the data and finalize the paper, and now that it is published, to our knowledge, it has not led to any formal change regarding PSMA PET/CT authorization.

In conclusion, the manuscript was interesting, and we especially acknowledge the interest of ANSM in promoting a survey to evaluate a new tracer. However, a closer collaboration with scientific societies could be beneficial to design better and less biased studies. EAU and EANM would have been relevant candidates for such a purpose.

\section{Declarations}

Ethical approval Not applicable.

Informed consent Not applicable.

Conflict of interest The authors declare no conflict of interest.

\section{References}

1. Chevalme YM, Boudali L, Gauthé M, et al. Survey by the French Medicine Agency (ANSM) of the imaging protocol, detection rate, and safety of 68 Ga-PSMA-11 PET/CT in the biochemical recurrence of prostate cancer in case of negative or equivocal $18 \mathrm{~F}$ fluorocholine PET/CT: 1084 examinations. Eur J Nucl Med Mol Imaging. 2021. https://doi.org/10.1007/s00259-020-05086-1 Online ahead of print.

2. Czernin J. The National Oncologic PET Registry (NOPR): a monumental effort by a few leaders. J Nucl Med. 2018;59(3):379.

3. Hillner BE, Hanna L, Makineni R, et al. Intended Versus Inferred Treatment After 18F-Fluoride PET Performed for Evaluation of Osseous Metastatic Disease in the National Oncologic PET Registry. J Nucl Med. 2018;59(3):421-6.

4. Nanni C, Zanoni L, Pultrone C, et al: (18)F-FACBC (anti1-amino-3(18)F-fluorocyclobutane-1-carboxylic acid) versus (11)C-choline PET/CT in prostate cancer relapse: results of a prospective trial. Eur J Nucl Med Mol Imaging 2016, 43 (9): 1601-1610.

Publisher's note Springer Nature remains neutral with regard to jurisdictional claims in published maps and institutional affiliations. 